\title{
Implementation of an Educational Platform on Power Quality
}

\author{
Aurora Gil-de-Castro, Isabel M. Moreno-Garcia, Victor Pallares-Lopez, David Matabuena, \\ Ricardo Medina-Gracia and Antonio Moreno-Munoz \\ Departamento de Ingeniería Electrónica y Computadores \\ Universidad de Córdoba, Campus de Rabanales, Edificio Leonardo Da Vinci, E-14071 Córdoba, España \\ agil@uco.es
}

\begin{abstract}
This paper describes a laboratory setup that allows students to gain knowledge in Electrical Engineering and Electronics. In this paper, the developed lab for power quality (PQ) monitoring is described. The set is implemented using the National Instrument (NI) Educational Laboratory Virtual Instrumentation Suite (ELVIS) and custom-made load boards. The software is based on the NI LabVIEW development environment. The set has been developed and employed for teaching electronic and instrumentation courses within the Master's degree in Distributed Renewable Energies from the University of Cordoba. The paper shows different practical lessons that are designed to perform typical PQ measurements such as power, frequency, RMS or THD.
\end{abstract}

Keywords - Engineering education, power quality, virtual instrumentation.

\section{INTRODUCTION}

Electrical and electronic engineering education is based on practical work which complements the theoretical teaching. However, not always is possible to take those practical lessons, as the high number of students attending courses, as well as the discipline itself, make that not all the subjects are prone to develop real practical lessons. Therefore, educators need to re-conceptualize fundamental issues of teaching, learning and assessment in nontraditional spaces. To integrate this new educational approach and the adaptation of embedded systems in the remotely controlled laboratory is therefore a challenge.

One example is found in [1], which aim is to establish a real-time web-based laboratory platform, able to access the laboratory set-up from a remote location. It uses LabVIEW (LABoratory Virtual Instrumentation Engineering Workbench), as is one of the primary choices in designing control and analysis solutions virtually and/or remotely in the engineering technology and education area.

A complete platform to understand power quality (PQ) concepts and analyze the characteristics of the power distribution network has been developed. The platform has been used with students enrolled in the Master's degree in Distributed Renewable Energies from the University of Cordoba

(https://www.uco.es/estudios/idep/masteres/energias-

This work has been supported by the Spanish Ministry of Economy and Competitiveness under Project TEC2016-77632-C3-2-R. Also, the authors would like to thank the support of the University of Cordoba through the program "III Becas Semillero de Investigación". renovables-distribuidas). More specifically, in the subject entitled Quality and Reliability of the Power Grid, within the above Master's Degree, the network is studied from the PQ point of view, including the origin and effects of different disturbances [2].

The system that students use for those practical lessons includes three major components: 1) PC equipped with the LabVIEW and custom-developed application-specific software, 2) National Instruments Educational Laboratory Virtual Instrumentation Suite (NI ELVIS) engineering lab workstation and a Data Acquisition (DAQ) module, and 3 ) a custom-designed integrated circuit (IC) testing load board. The advantage of using this programming language is that it is easy to use, and students can rapidly interface with measurement and control hardware, and analyze data.

For each system, the students are required to learn the underpinning theoretical and practical test engineering study materials, develop and implement the LabVIEW-based application specific software and graphic user interface (GUI) so to enable the system to perform the required test types automatically.

The aim of the proposed exercises is to easily understand PQ basic parameters by students. So that, a resistor as well as a series resistor and inductance loads are connected to the grid and the different magnitudes are calculated. Active, reactive and apparent power, energy, as well as magnitude and frequency and harmonics are calculated. The evaluation of the practical lesson consists on a summary of the main results, as well as the code in LabVIEW.

The paper is organized as follows. Section 2 describes the materials used in the practical lessons, including hardware and software. Section 3 presents the implemented power quality disturbances, including Power and Energy, Magnitude and Frequency, Harmonic and Unbalance. Section 4 includes the final implementation. Finally, Section 5 contains the conclusions.

\section{MATERIALS}

\section{A. Hardware}

The NI ELVIS Engineering Lab Workstation provides a complete teaching solution for engaging students in hands-on 
labs involving areas such as analog circuits, power electronics, and instrumentations among others. It includes 12 softwarebased virtual instruments including an oscilloscope, digital multimeter, function generator, variable power supply, and Bode analyzer [3].

The NI ELVIS has 8 differentials or 16 single ended channels. In the proposed practical lesson, students have connected voltage and current transducers, manufactured by the University as part of a Final Master Project, to the acquisition board in order to adapt voltage levels to the input of the NI ELVIS.

The voltage (LEM LV 25-P [4]) and current (Honeywell 0730 [5] transducers are used to measure AC current with galvanic separation between the primary and secondary circuit. According to the voltage transducer datasheet, a voltage of up to $500 \mathrm{~V}$ can be measured, and a $40 \mathrm{k} \Omega$ was selected as R1 to transform the AC voltage in a nominal current of $10 \mathrm{~mA}$ at full scale. At the output block, a high precision resistor was selected in order to transform the current into a voltage within the range of the acquisition card $\left(\mathrm{R}_{\mathrm{M}}\right.$ in Fig. 1).

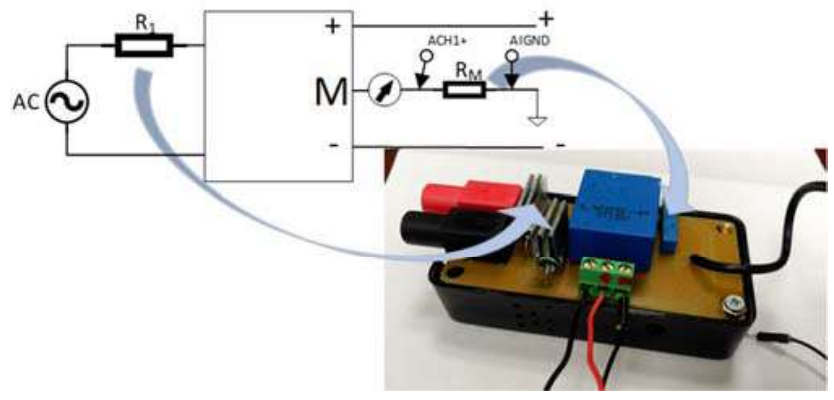

Fig. 1. Signal Conditioning of the DC and AC voltage transducers.

For current measurement, a similar principal schema is used. A conditioning of current measurements was needed to adjust the levels for the data acquisition card. Fig. 2 shows an example of the conditioning circuit of the developed current transducer and the real implementation. In this case, the output range is selected and calibrated using multi-turn precision resistors, labelled as $R_{M}$ in the figure.

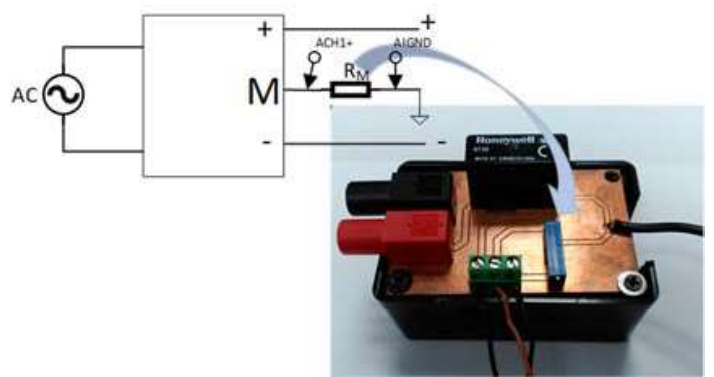

Fig. 2. Signal Conditioning of the continuous (DC) and alternating (AC) current transducers.

The NI ELVIS was used because students can easily test the circuit using the integrated oscilloscope and multimeters. Voltage and current waveforms are easily seen in the integrated oscilloscope, as well as measurements as active power are easily performed with the integrated multimeter. But the big advantage comes from the acquisition card, as student can understand the voltage and current processing, as well as the power quality concepts from a mathematical point of view. The aim of the practical lesson is to calculate the harmonic spectrum, power, energy, displacement power factor, and some other parameters using the information from the voltage and current waveform vectors.

The whole platform is then connected to a computer where the signals are shown. The final implementation is then described in section 4 .

\section{B. Software}

The PQ analysis data acquisition was performed through virtual instruments (VI) developed with the NI LabVIEW graphical programming tool and operating in a computer connected to NI ELVIS platform. Students are provided by a LabVIEW project (downloaded from Moodle platform http://moodle.uco.es/m1718/) with four VIs, each of them focused to different PQ aspects: energy and power, magnitude and frequency, harmonics, and unbalance. Each of them will later be described in section III. The first step before indeed using each VI is focused in explaining the importance of knowing the corresponding magnitude in the PQ analysis, including the definition, the source of occurrence, and the mitigation procedures. Then, the Toolkit functions that can be used for that magnitude are explained. Later, the procedure to include such function in the corresponding VI is described, while students are implementing that function into their own VI. Eventually, the student must run the library and analyse the monitored signals.

$\mathrm{AC}$ voltages and currents measurements were acquired according to several electromagnetic compatibility standards, such as IEC 61000-4-7:2002 [6], IEC 61000-4-15:2010 [7], and IEC 61000-4-30:2008 [8]. This processing was possible through the Electrical Power Suite Toolkit, an extra resource not licensed by LabVIEW. This Toolkit includes a collection of tools for measuring, analyzing, monitoring, and recording electrical power data.

\section{IMPLEMENTED POWER QUALITY DISTURBANCES}

The aim of the practical lesson is to experimentally analyse PQ magnitudes. The general methodology used for the development of the lesson consists in a student-guided integration of the functions contained in the LabVIEW Electrical Power Toolkit to explain students the general operation. To do that, the responsible of the subject has an identical characteristic working place than students has. The code is projected in the screen while the integration of the different functions to be used is explained step by step.

Before working with real signals, the practical lesson is prepared to make students work with synthetical current and voltage signals simulated by themselves. The aim is allowing students to emulate different PQ disturbances, so that they can study and check the efficiency of the libraries to be used. Fig. 3 shows the main VI panel that allows student configuring the synthetical signals. 


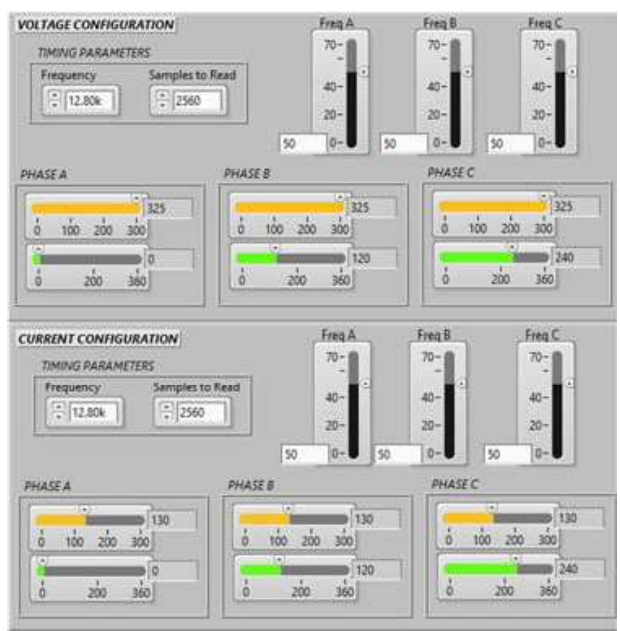

Fig. 3. Voltage and current configuration panel.

In the upper part of Fig. 3 the frequencies can be adjusted, individually for each phase. Below the peak value and phase angle with respect to A phase can be introduced. Moreover, as they are synthetical signals, three-phase current configuration is also possible. Similar to the voltage waveforms, frequency, peak value and phase angle of the three phases can be modified.

There is another panel where students can indeed check the waveforms. The three-phase voltage and current waveforms are shown in Fig. 4. Both, Fig. 3 and 4 are provided to the students for testing the Toolkits about power and energy, magnitude, frequency and unbalance. The starting VI for the harmonic measurement is different from the previous three cases and will be explained in section C.

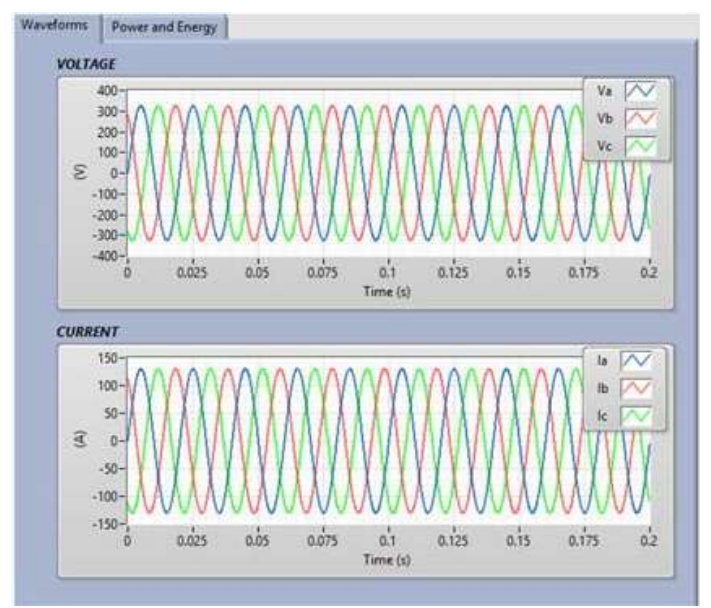

Fig. 4. Voltage and current waveforms visualization panel.

\section{A. Power and Energy}

In this test the students must generate different voltage and current waveforms, and measure power and energy values of those signals with the Power and Energy VIs. Power VI returns power values that this VI calculates through a basic measurement time interval of 10 cycles. The students calculate for each phase power values from voltage and current spectra (Fig. 5 left). These values are active power; reactive power; apparent power; fundamental active power; fundamental reactive power; cosine; power factor.

With the Energy VI, the students calculate energy values for a specified interval length established in one hour (Fig. 5 right). This VI calculates the energy values from the data provided in the Power VI. These values are active energy; reactive energy; apparent energy; fundamental active energy; fundamental reactive energy; active positive energy; active negative energy; reactive inductive energy; reactive capacitive energy.

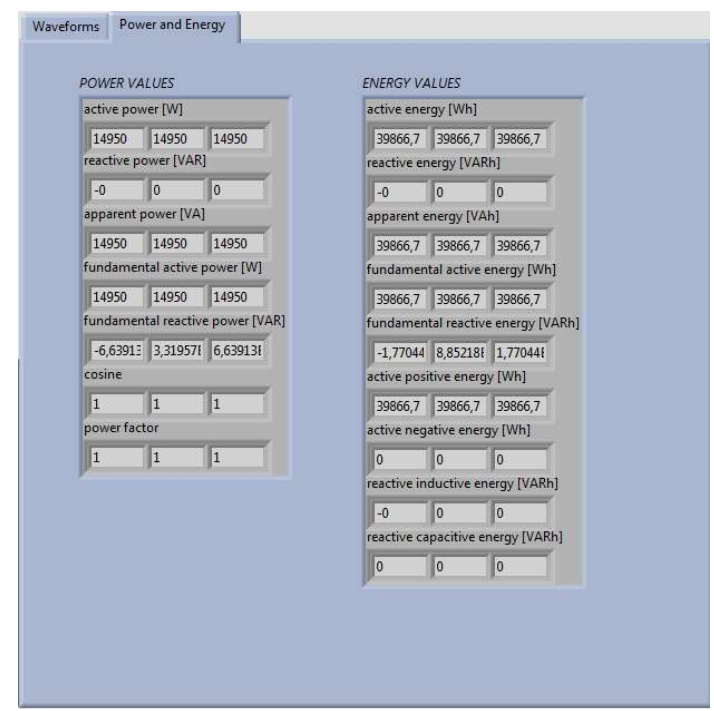

Fig. 5. Power and energy panel

\section{B. Voltage and current magnitudes and Frequency}

The aim of this part of the practical lesson is to calculate voltage and current RMS values, overvoltage and undervoltage, as well as measure frequency using the Zerocrossing function. To do that, students analyze their RMS value, calculating mean value, maximum and minimum deviation.

Both for voltage and current, firstly they use the RMS VI which returns RMS value of the magnitudes for a 10-cycle block for three- phases. Secondly, after calculating the RMS value, students calculate underdeviation and overdeviation for each voltage phase with the Deviation VI.

Finally, students use the Frequency VI to calculate the average frequency using zero crossing function. The frequency is only calculated over one phase.

As can be seen in Fig. 6, the instantaneous frequency values, RMS values of three-phase voltages and currents are shown in graphs to observe their trends. While under and over values are shown in numerical indicators. In the displayed example (Fig. 6) the A-phase frequency, the B-phase voltage amplitude and the $\mathrm{C}$-phase current amplitude were varied. 


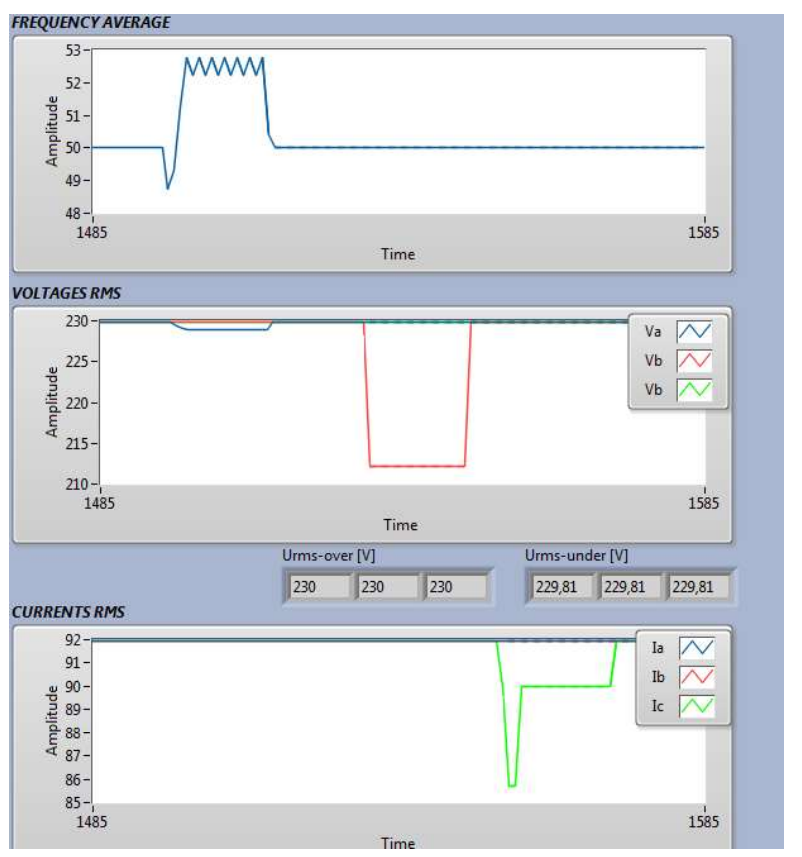

Fig. 6. Measurements panel.

\section{Harmonic}

In this section, the aim was to calculate the total harmonic distortion (THD) of the voltage and current, compute the harmonics and interharmonics according to the grouping method, and add different harmonic amplitudes to the fundamental to analyze results from that. The harmonics VI is used to perform harmonics analysis according to IEC 610004-7:2002 [6] and IEC 61000-4-30:2008 [8].

To test waveforms containing harmonic distortion, the addition of harmonics to the fundamental voltage waveform is included in the voltage configuration panel. For each phase and each harmonic order, the level (in percent of fundamental) as well as the phase angle shift to the corresponding fundamental can be added (Fig. 7). In this case, the phase A has a $50 \%$ FND of harmonic 3 , and $20 \%$ of FND of harmonic 9 , both with $0^{\circ}$ phase angle.

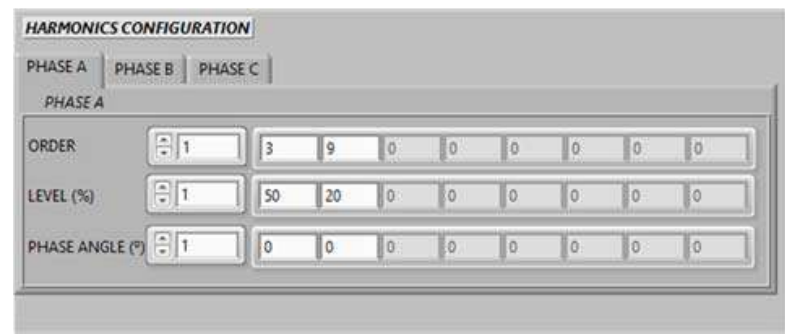

Fig. 7. Harmonics configuration panel.

When the magnitude and phase angle of different harmonic orders are added to the fundamental, the resulted distorted voltage waveform is shown in the corresponding Phase A (B or $\mathrm{C}$ according to the phase) tab. Fig. 8 above (equal in each phase) shows the fundamental voltage, and below is shown the resultant voltage waveform after adding $3^{\text {rd }}$ and $9^{\text {th }}$ harmonic orders to the fundamental. In the waveforms tabs appears the three-phase voltage and current (similar to Fig. 4).

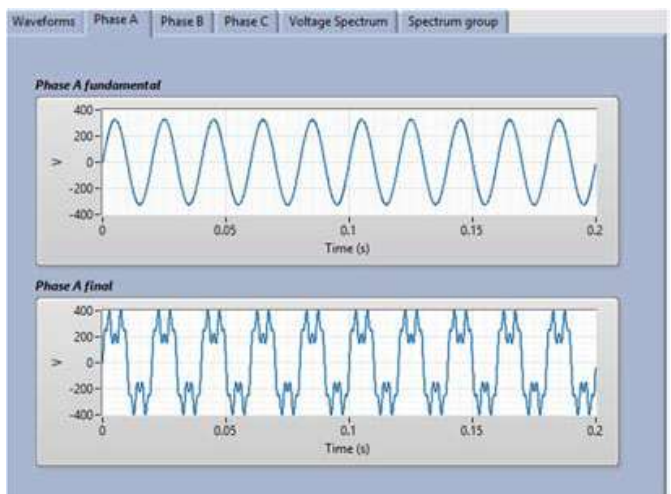

Fig. 8. Distorted voltage waveforms panel

To contrast those results, the voltage spectrum is used to compute the FFT spectra of input voltage waveforms. This VI returns FFT spectra as complex spectra of voltage, up to $50^{\text {th }}$ order $(2500 \mathrm{~Hz}$ ) with a frequency resolution around $5 \mathrm{~Hz}$ (over 10 -cycle window) in RMS. Fig. 9 above shows for phase A the $3^{\text {rd }}(150 \mathrm{~Hz})$ and $9^{\text {th }}(450 \mathrm{~Hz})$ harmonic orders with $50 \%$ of FND $(115 \mathrm{~V})$ and $20 \%$ of FND (46 V) respectively with a 230 VRMS fundamental voltage. And for phase $B$ the $5^{\text {th }}(250$ $\mathrm{Hz})$ and $7^{\text {th }}(350 \mathrm{~Hz})$ harmonic orders with $30 \%$ of FND (69 $\mathrm{V})$ and $10 \%$ of FND $(23 \mathrm{~V})$ respectively.

The voltage THD is calculated according to IEC 61000-4$7: 2002$ [6]. This VI calculates the group THD (up to the $40^{\text {th }}$ order), expressed in percentage for each phase. Fig. 9 below shows $53.8 \%$ in phase $A$ and $31.6 \%$ in phase B.

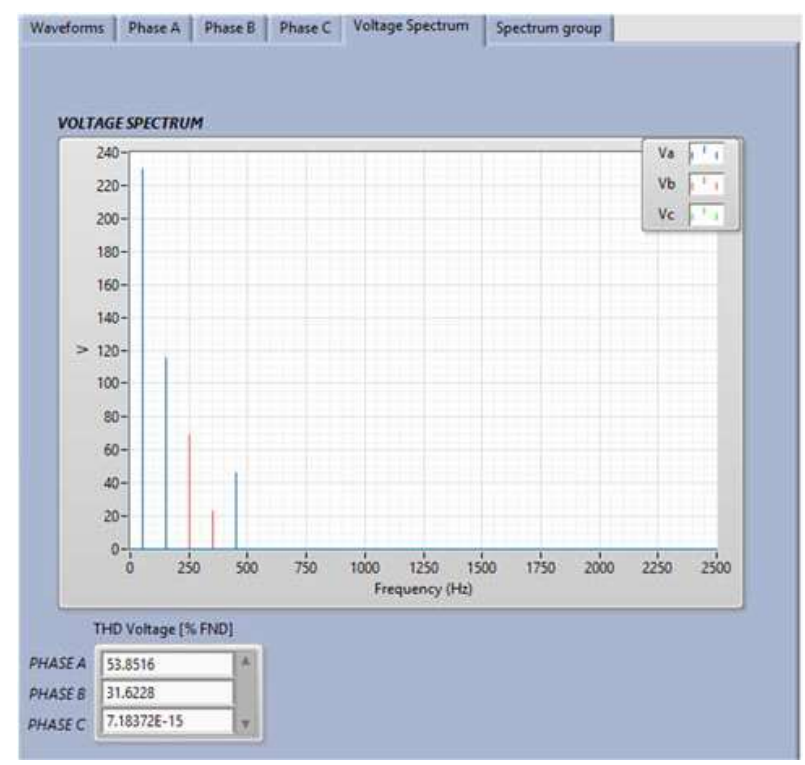

Fig. 9. Voltage spectrum panel.

\section{Unbalance}

This test is intended for students to implement the code to measure unbalance factors and symmetrical components, display the voltage and current diagram. To do this, they must 
use the following library functions: Symmetrical Components VI and Unbalance VI.

The Symmetrical Components and Unbalance VIs are used to calculate symmetrical components and analyze voltage and current unbalance for a three-phase electrical power system, as defined in IEC 61000-4-30:2008 [8].

On the one hand, Symmetrical Components VI calculates voltage, current, and power symmetrical components for a 10cycle block. This VI is used to calculate the fundamental voltage symmetrical components. All the symmetrical components have the following array order: zero, positive and negative sequence symmetrical components

On the other hand, the Unbalance VI is useful to analyze the voltage or current unbalance of a three-phase electrical power system. This VI is used to calculate negative sequence (u2) and zero sequence (u0) expressed as a percentage of voltage unbalance, fundamental voltage unbalance, percentage of current unbalance, and fundamental current unbalance.

The three-phase voltage and current polar plot is also shown in Fig. 10 (below). In the test shown in Fig. 10, an unbalance in the three-phase voltage signal was configured through a voltage sag in phase B and a phase angle shift in phase C. Similarly, an unbalance in the three-phase voltage signal was configured through a voltage sag in phase $\mathrm{C}$ and a phase angle shift in phase B.

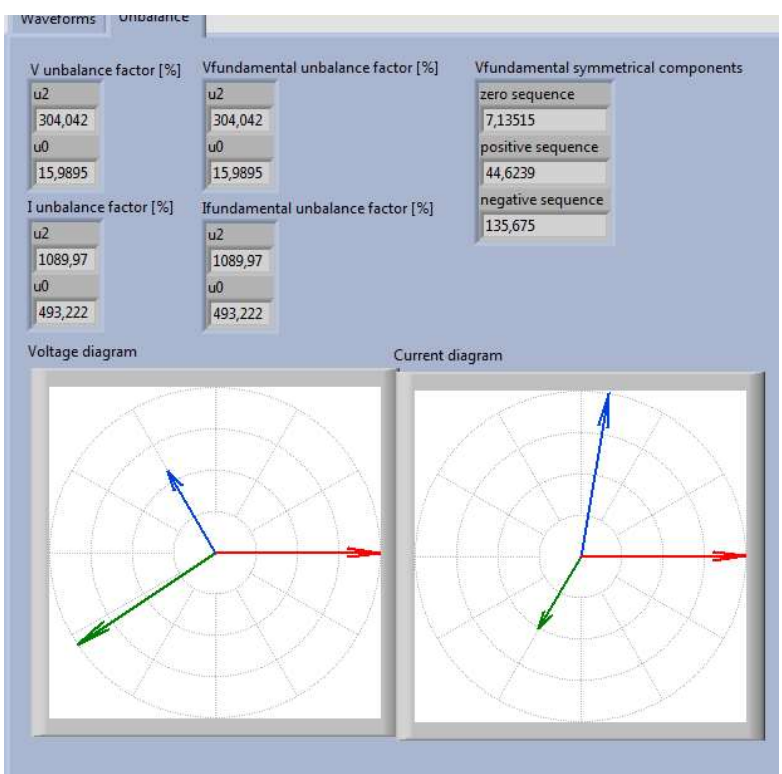

Fig. 10. Distorted voltage waveforms panel.

\section{FINAL IMPLEMENTATION}

The NI ELVIS and the transducers are used to analyze PQ data over different loads. To do so, voltage and current are recorded when different loads as a $500 \Omega$ load, and a series resistor and inductance load are connected to the grid. Finally, students calculate different magnitudes over that signals. Active, reactive and apparent power, energy, as well as magnitude and frequency and harmonics are calculated, and those values are asked to students as a part of the evaluation.

Fig. 12 shows the final implementation that students should perform by the end of the session. It must integrate all the measurements included in section III in a single VI project (Fig. 11), connect the loads to the acquisition system, and generate different disturbances.

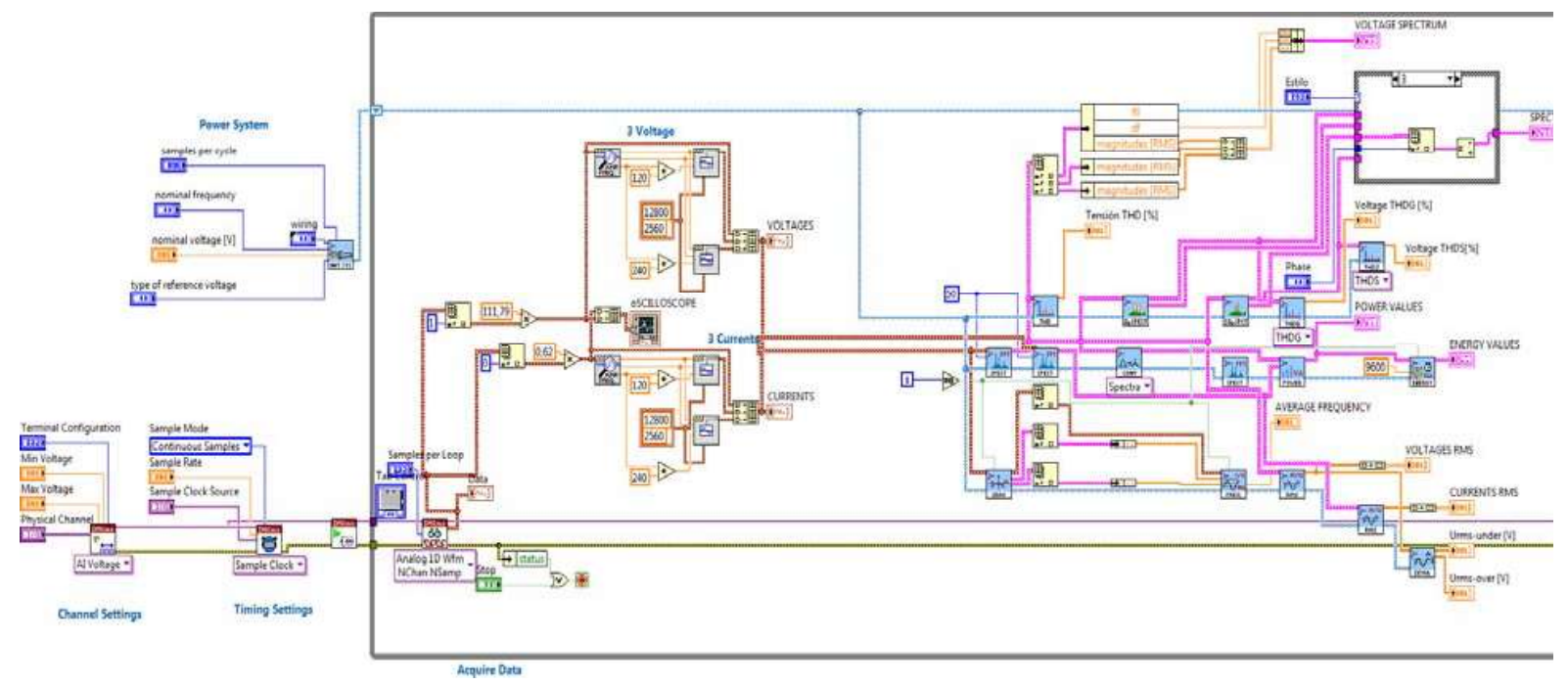

Fig. 11. LabVIEW final code. 


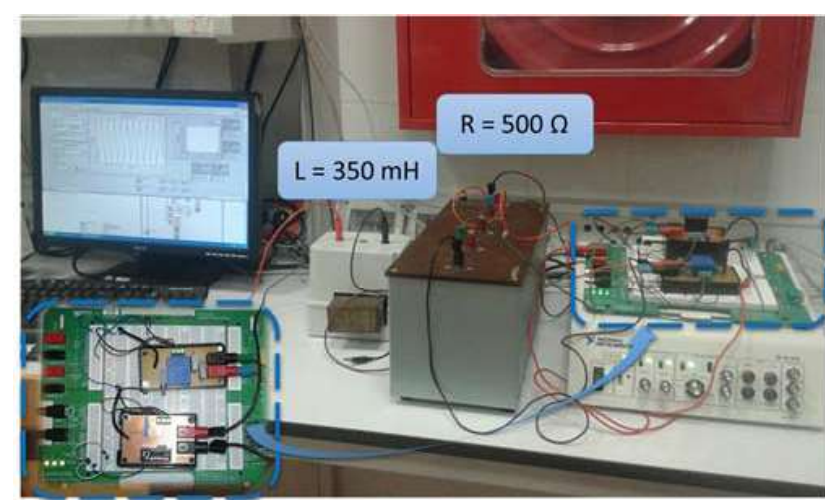

Fig. 12. Final implementation

Students are also provided with a table to be filled with the different connected loads. As an example, Table I contains the demanded data when connected to a resistor-inductance series load.

TABLE I. DATA TO MEASURE

\begin{tabular}{|l|c|c|c|}
\hline \multicolumn{3}{|c|}{ Resistive and inductance series load } \\
\hline \multicolumn{1}{|c|}{ Data } & Value & Data & Value \\
\hline Active power & & Average frequency & \\
\hline Reactive power & & V RMS & \\
\hline Aparent power active & & V RMS over & \\
\hline $\begin{array}{l}\text { Fundamental } \\
\text { power }\end{array}$ & & THD under & \\
\hline $\begin{array}{l}\text { Fundamental reactive } \\
\text { power }\end{array}$ & & THD grouping & \\
\hline $\begin{array}{l}\text { Displacement power } \\
\text { factor }(\cos \varphi)\end{array}$ & & THD subgrouping & \\
\hline \begin{tabular}{l} 
Total power factor \\
\hline
\end{tabular} & & & \\
\hline
\end{tabular}

\section{CONCLUSIONS}

The aim of the proposed exercises is to easily understand PQ basic parameters by students. Active, reactive and apparent power, energy, as well as magnitude and frequency and harmonics are calculated. The evaluation of the practical lesson consists on a summary of the main results, as well as the code in LabVIEW.

Some of the key point of the tool is that includes different learning approaches such as exploratory learning and inquirybased learning, as students develop a tool based in a preliminary version, and they can check the proper working of the platform. The tool presents interactivity, which makes the tool more attractive, as well as use elements of multimedia for visualisation and direct analysis.

Students appreciate these practical lessons to better understand such a deep field as PQ is. A satisfaction questionnaire was carried out between master degree students to evaluate the usefulness of the platform. Such a tool was positively evaluated, and the platform efficiency in the learning process showed a final examination main score of 4.5 out of 5 points.

\section{REFERENCES}

[1] A. Akar, A. Yayla, and B. Erder, "A labVIEW-based remotely controllable and accessible laboratory platform for communication systems II lecture experiments," Gazi University Journal of Science, vol. 27, pp. 965-977, 2014.

[2] M. T. Chew, S. Demidenko, M. P. L. Ooi, and Y. C. Kuang, "Family of low-cost NI ELVIS/LabVIEWbased semiconductor testers for engineering education," in 2017 IEEE International Conference on Computational Intelligence and Virtual Environments for Measurement Systems and Applications, CIVEMSA 2017 - Proceedings, 2017, pp. 165-170.

[3] N. ELVIS. Available: http://www.ni.com/pdf/productflyers/ni-elvis.pdf

[4] L. L. 25-P. Available at: http://www.lem.com/docs/products/lv 25-p.pdf

[5] H. $0730 . \quad$ Available at: https://sensing.honeywell.com/honeywell-sensingcurrent-csn-series-catalog-pages.pdf

[6] "Electromagnetic compatibility (EMC) - Part 4-7: Testing and measurement techniques - General guide on harmonics and interharmonics measurements and instrumentation, for power supply systems and equipment connected thereto, IEC 61000-4-7, August 2002.

[7] I. E. Commission, "Electromagnetic compatibility (EMC) - Part 4-15: Testing and measurement techniques - Flickermeter - Functional and design specifications, IEC 61000-4-15, 2010.

[8] "Electromagnetic compatibility (EMC) - Part 4-30: Testing and measurement techniques - Power quality measurement methods, IEC 61000-4-30, October 2008. 